\title{
Clinical analysis of the prognosis after receiving a liver graft that abandoned transplantation due to poor graft conditions in the centers allocated as a priority
}

\author{
Ho Joong Choi, Changho Seo, Sung Eun Park, Joseph Ahn, Tae Ho Hong, Young Kyoung You
}

Division of Hepatobiliary, Department of Surgery, Seoul St. Mary's Hospital, College of Medicine, The Catholic University of Korea, Seoul, Korea

Background: Liver transplantation (LT) has the limitation of lack of donors compared to those waiting for a transplant. Depending on the recipient's condition, even liver grafts with poor conditions may need to be transplanted. This study was conducted to analyze the prognosis after LT that abandoned transplantation due to poor graft conditions at the preceding centers.

Methods: From January 2010 to September 2020, deceased-donor LT was performed in 161 patients in Seoul St. Mary's Hospital. Among them, 127 patients (allocated group) were preferentially allocated to our center by Korean Network for Organ Sharing and the remaining 34 patients ( $21.1 \%$, abandoned group) received liver grafts that were abandoned by other transplant centers due to poor organ conditions. To compare and analyze the clinical prognosis of the allocated group and the abandoned group, various perioperative factors and postoperative outcomes were evaluated.

Results: The average ages of the allocated and abandoned groups were 52.3 and 51.6 years, respectively $(P=0.65)$. The preoperative Model for End-stage Liver Disease score was $27.3 \pm 10.6$ in the allocation group and $25.8 \pm 11.3$ in the abandoned group $(P=0.49)$. There was no difference between the two groups in operation time $(P=0.14)$ and intraoperative $P R C$ transfusion $(P=0.94)$. There was no difference between the two groups in intensive care unit stay $(P=0.91)$ and hospital stay $(P=0.86)$. In-hospital mortality occurred in 17 patients (13.4\%) in the allocated group and three patients (8.8\%) in the abandoned group, so there was no difference between the two groups $(P=0.47)$. The 5 -year survival rate was $72.4 \%$ in the allocated group and $78.2 \%$ in the abandoned group, with no difference between the two groups $(P=0.25)$.

Conclusions: Even if the graft that was abandoned due to poor condition, good results can be obtained if the transplant is carried out according to the recipient state. And as a result, it is expected that the discarded graft can be reduced. 